\title{
Spike-timing dependent plasticity beyond synapse - pre- and post-synaptic plasticity of intrinsic neuronal excitability
}

\author{
Dominique Debanne ${ }^{1,2 *}$ and Mu-Ming Poo ${ }^{3}$ \\ Institut National de la Santé et de la Recherche Médicale Unité 641, Marseille, France \\ Faculté de Médecine secteur nord, Université de la Méditerranée, Marseille, France \\ ${ }^{3}$ Department of Molecular and Cell Biology, Division of Neurobiology, University of California, Berkeley, CA, USA
}

\section{Edited by:}

Henry Markram, Ecole Polytechnique

Federale de Lausanne, Switzerland

\section{Reviewed by:}

Eve Marder, Brandeis University, USA Henry Markram, Ecole Polytechnique

Federale de Lausanne, Switzerland

\section{*Correspondence:}

Dominique Debanne, Institut National de la Santé et de la Recherche Médicale Unité 641, Faculté de

Médecine secteur nord, Boulevard

Pierre, Dramard 13344, Marseille,

France.

e-mail:dominique.debanne@univmed.fr
Long-lasting plasticity of synaptic transmission is classically thought to be the cellular substrate for information storage in the brain. Recent data indicate however that it is not the whole story and persistent changes in the intrinsic neuronal excitability have been shown to occur in parallel to the induction of long-term synaptic modifications. This form of plasticity depends on the regulation of voltage-gated ion channels. Here we review the experimental evidence for plasticity of neuronal excitability induced at pre- or postsynaptic sites when long-term plasticity of synaptic transmission is induced with Spike-Timing Dependent Plasticity (STDP) protocols. We describe the induction and expression mechanisms of the induced changes in excitability. Finally, the functional synergy between synaptic and non-synaptic plasticity and their spatial extent are discussed.

Keywords: hippocampus, cortex, LTP, LTD, ion channel, plasticity, STDP

\section{INTRODUCTION}

Long-lasting plasticity of synaptic transmission was for years considered as the favorite mechanism to account for information storage in the brain. Bidirectional long-term synaptic plasticity is indeed computationally appealing, principally because of synapse-specific changes among a large array of inputs. However, this is not the whole story and many additional electrophysiological components of neurons may undergo use-dependent long-term plasticity (Kim and Linden, 2007). Besides electrical synapses and glutamate transporters, voltage-gated ion channels occupy a key position in the persistent filtering of the neuronal message (Sjöström et al., 2008).

\section{PLASTICITY OF INTRINSIC NEURONAL EXCITABILITY: A CELLULAR MECHANISM OF LEARNING AND MEMORY}

The postulate that modification in intrinsic excitability could underlie the formation of functional neuronal assemblies and may thus contribute to a specific memory trace has its origin in invertebrate neural systems. The first evidence for intrinsic plasticity came from the pioneering work of Alkon who showed that phototaxic learning in the marine mollusk Hermissenda crassicornis involved the reduction of voltage-gated A-type and $\mathrm{Ca}^{2+}$-activated $\mathrm{K}^{+}$currents in a photoreceptor (Alkon, 1984). Several forms of learning in Aplysia (sensitization and operant conditioning) were also found to be associated with persistent changed in neuronal excitability (Scholz and Byrne, 1987; reviewed in Mozzachiodi and Byrne, 2010). Interest in plasticity of intrinsic excitability of mammalian neurons was for a long-time occluded by the challenge of dissecting the mechanisms underlying long-term synaptic potentiation (LTP) and depression (LTD). A new interest in the study of intrinsic plasticity in mammalian brain has flourished only during the 1990s. The search for cellular excitability corre- lates of learning and memory in the mammalian brain has focused on neurons that are specifically active during learning. Eyeblink conditioning in the cat or the rabbit is well documented in this respect since neurons from active circuits during the conditioning exhibit in vitro an excitability that is significantly elevated (Disterhoft et al., 1986; Aou et al., 1992; Schreurs et al., 1997; Matthews et al., 2008). In several cases, the after-hyperpolarization (AHP) current was found to be depressed. Other forms of learning such as spatial or olfactory learning involve the regulation of neuronal excitability (Saar et al., 1998; Oh et al., 2003). These observations therefore suggest that the memory trace could not only be supported by selective changes in synaptic strength but that modifications in neuronal excitability might also contribute to the cellular substrate of the memory trace in the brain. This conclusion must be however qualified since in hippocampal and cortical neurons, increased neuronal excitability is no longer detectable 8 days after eyeblink conditioning, although learning is still established (Moyer et al., 1996). These in vivo studies, therefore suggest that synaptic or propagated activity during the induction phase may determine the induction of intrinsic plasticity. However, several key questions regarding the induction and expression mechanisms of intrinsic plasticity cannot be satisfactorily addressed at a cellular and molecular scale in the in vivo preparation and the use of in vitro assays must be envisaged to accurately control synaptic activity, membrane potential as well as pharmacologically isolated membrane currents.

In central cortical neurons, excitatory postsynaptic potentials (EPSPs) measured at the axon hillock result from a tight interplay between synaptic and intrinsic voltage-gated conductances that either amplify or attenuate excitatory synaptic potentials (review in Spruston, 2008). Any modifications in this fragile equilibrium may in turn facilitate or diminish the probability that a given 
synaptic input triggers an action potential. Thus, regulation of ion channel activity represents a powerful means to control the principal neuronal output. For instance, tetanic stimulation of glutamatergic synaptic inputs not only induces LTP in hippocampal neurons but also persistently facilitates the generation of a postsynaptic action potential by the EPSP (Bliss et al., 1973; Andersen et al., 1980), a phenomenon known as EPSP-Spike potentiation. Notably, this form of plasticity is also observed in the presence of blockers of synaptic inhibition and is bidirectional (reviewed in Daoudal and Debanne, 2003). Induction of LTD with low frequency stimulation of the Schaffer collateral-CA1 neuron input is associated with a long-lasting decrease in EPSPSpike coupling (Daoudal et al., 2002). The three major classes of glutamate receptors (i.e., $N$-methyl-D-aspartate (NMDAR), metabotropic glutamate (mGluR) and kainate (KR) receptors) play a central role in the induction of long-lasting plasticity of intrinsic neuronal excitability. Synaptic activation of NMDAR induces a wide range of pre- and postsynaptic plasticities of neuronal excitability in hippocampal, neocortical and cerebellar neurons (Aizenman and Linden, 2000; Armano et al., 2000; Ganguly et al., 2000; Wang et al., 2003; Li et al., 2004; Fan et al., 2005; Kim et al., 2007; Campanac et al., 2008; Losonczy et al., 2008). And many different voltage-gated sodium, potassium and cationic currents are targeted by the stimulation NMDAR. Activation of mGluR also produces many forms of short- and long-term regulation of intrinsic excitability in hippocampal and neocortical and modulate calcium-activated potassium, voltage-gated sodium, and hyperpolarization-activated cationic channels (Ireland and Abraham, 2002; Sourdet et al., 2003; Carlier et al., 2006; Brager and Johnston, 2007). Synaptic activation of KR induces long-term increase in hippocampal neuron excitability that is mediated by a reduction of the slow calcium-activated potassium current $I_{\text {sAHP }}$ (Melyan et al., 2004; Ruiz et al., 2005).

Activation of glutamate receptors is not the only means to induce long-term plasticity of intrinsic excitability in central neurons. Postsynaptic spiking may also induce persistent increases in neuronal excitability of cerebellar and cortical neurons in vitro and in vivo (Aizenman and Linden, 2000; Egorov et al., 2002; Nelson et al., 2003; Cudmore and Turrigiano, 2004; Paz et al., 2009). In most of these cases, the cellular conditioning consists of repeated postsynaptic bursting induced by direct injection of depolarizing current in the neuron that produce a postsynaptic elevation in $\mathrm{Ca}^{2+}$ concentration, and the subsequent activation of protein kinases and the regulation of voltage-gated ion channels.

\section{SPIKE-TIMING DEPENDENT PLASTICITY}

Spike-timing dependent plasticity (STDP) has originally been defined for excitatory synaptic transmission in hippocampal and neocortical circuits (Levy and Steward, 1983; Gustafsson et al., 1987; Debanne et al., 1994, 1996, 1998; Markram et al., 1997; Bi and Poo, 1998; Feldman, 2000; Sjöström et al., 2001; reviewed in Abbott and Nelson, 2000; Dan and Poo, 2006). Synaptic efficacy is persistently and bi-directionally altered depending on the temporal interaction between afferent synaptic activity (EPSP) and postsynaptic spiking (the action potential). In most excitatory synapses positively correlated pre- and postsynaptic activity (EPSP before action potential) leads to synaptic reinforcement whereas negative correlation (action potential before EPSP) induces LTD. Thus, as initially postulated by Hebb (1949), synapses that repeatedly contribute to the postsynaptic discharge are reinforced whereas synapses that repeatedly fails to participate to the neuronal activity are depressed (Stent, 1973). Since the early 1990's, this rule has been verified in a large number of glutamatergic synapses impinging on glutamate neurons (Gustafsson et al., 1987; Debanne et al., 1994, 1996, 1998; Markram et al., 1997; Bi and Poo, 1998; Tzounopoulos et al., 2004; but see Egger et al., 1999). However, when the postsynaptic neuron is inhibitory this rule is generally inverted (Bell et al., 1997; Wang et al., 2000; Tzounopoulos et al., 2004; Fino et al., 2005; but see Pawlak and Kerr, 2008). A major consequence of STDP is that synaptic inputs compete for postsynaptic spike-timing and short-latency inputs win over long-latency synapses (Song et al., 2000). STDP is also observed in many different in vivo preparations in which synaptic activity can be monitored in response to electrical or sensory stimulation (Zhang et al., 1998; Jacob et al., 2007). It may account for activity-dependent plasticity of orientation maps in the cat striate cortex (Schuett et al., 2001), sensory adaptation (Yao and Dan, 2001) or shaping of hippocampal place-fields in the rat (Mehta et al., 2000). STDP rules have also been verified in the human brain with peripheral and transcranial stimulation (Wolters et al., 2003). However, when functional plasticity is monitored in vivo, it is virtually impossible to precisely identify the neuronal substrate and the underlying mechanisms of the observed changes. They may, indeed, equally result from modification of either synaptic strength or intrinsic excitability, or both. Therefore, it is of great importance to know whether protocols based on temporal interactions also produce long-lasting modifications of neuronal excitability in hippocampal and cortical neurons, and the spatial and temporal extent of these modifications.

\section{PRESYNAPTIC CHANGES IN NEURONAL EXCITABILITY AND STDP}

Changes in neuronal excitability associated with synaptic plasticity induced by STDP protocols have first been identified at the presynaptic side. Using paired-recordings from synaptically coupled hippocampal neurons, Ganguly et al. (2000) found that correlated pre- and postsynaptic spiking not only induces LTP of synaptic transmission but also increases the excitability of the presynaptic neuron. This presynaptic plasticity of neuronal excitability is rapidly expressed ( $<2 \mathrm{~min})$ and is long-lasting (>30 min). Its induction requires activation of NMDA receptors (NMDAR), postsynaptic $\mathrm{Ca}^{2+}$ elevation and activation of protein kinases $\mathrm{C}(\mathrm{PKC})$. The expression mechanisms have been studied at the soma of the presynaptic neuron and involve a shift in the activation curve of the $\mathrm{Na}^{+}$current towards hyperpolarizing values that may account for the lower spike threshold (Ganguly et al., 2000). In addition, the sodium current exhibits an improved recovery from inactivation following correlated pre- and postsynaptic activity.

Mirror effects have been observed presynaptically when LTD is induced by negative correlation in hippocampal and neocortical pyramidal neurons (Li et al., 2004). The excitability of the presynaptic cell is persistently reduced following induction of LTD. This plasticity requires postsynaptic calcium elevation and 
presynaptic PKA- and PKC-dependent modification of slowactivating $\mathrm{K}^{+}$channels. The reduction in excitability is reversible if LTP is induced with positive correlation (Li et al., 2004). It is important to note here that these presynaptic changes in excitability were also observed in pairs of L4 spiny neurons from brain slices, suggesting that this form of intrinsic plasticity might also be present in vivo.

The functional consequences of long-lasting plasticity of presynaptic excitability might be of great importance for the dynamics of the neural circuits. For instance, the presynaptic increase in neuronal excitability following correlated spiking activity may facilitate the induction of bursting-like behavior and will enhance the reliability of signal transmission by creating privileged activity pathways in the brain. In addition, enhancement of presynaptic neuronal excitability may affect the plasticity of upstream synapses made onto the presynaptic cell by facilitating the initiation of backpropagated action potentials (Tao et al., 2000). Indeed, retrograde spread of LTP and LTD from developing retinotectal synapses to upstream synapses made by bipolar cells on retinal ganglion cells in the retina has been observed in vivo, through cytoplasmic retrograde signaling with the order of minutes after the induction of LTP/LTD (Du et al., 2009). Presynaptic modifications of neuronal excitability and efficacy of upstream synapses may be attributed to the actions of common retrograde signals and serve synergistic functions in neuronal circuit plasticity.

\section{POSTSYNAPTIC CHANGES IN NEURONAL EXCITABILITY AND STDP}

Neuronal excitability is also affected on the postsynaptic side when long-term synaptic plasticity is induced with STDP protocols. At least two neuronal functions, namely EPSP summation and EPSPspike coupling, are modulated after the induction of spike-timing dependent LTP/LTD, through changes in postsynaptic voltagegated channels.

In hippocampal CA1 pyramidal neurons, summation of EPSPs is determined by voltage-gated conductances that amplify or attenuate synaptic potentials. The property of EPSP summation in these neurons is strongly modulated following induction of LTP or LTD (Wang et al., 2003; Xu et al., 2006). A gain in EPSP summation is consistently observed when LTP is induced with positively correlated activity whereas a loss of summation is associated with LTD induced by negative correlation. The activity-dependent increase in EPSP summation requires activation of NMDA receptors, PKC and $\mathrm{Ca}^{2+} /$ calmodulin-dependent protein kinase II (CaMKII), but is independent of $\mathrm{GABA}_{\mathrm{A}}$ receptor-mediated inhibition (Wang et al., 2003). Interestingly, blockers of hyperpolarization-activated cationic (h) channels prevent the facilitation in EPSP summation, suggesting that the facilitation results from the down-regulation of h-channel activity in the dendrites.

EPSP-spike coupling is also profoundly affected in CA1 pyramidal neurons when long-term synaptic plasticity is induced with STDP protocols (Campanac and Debanne, 2008). Positive correlation between pre- and postsynaptic activity not only induces LTP but also facilitates EPSP-spike coupling. Conversely, LTD induced by negative correlation is associated with EPSP-spike depression. Both EPSP-spike potentiation and depression require activation of NMDA receptors but are independent of $\mathrm{GABA}_{\mathrm{A}}$ receptor dependent inhibition (Campanac and Debanne, 2008). The amplitude-slope relation of the EPSP was facilitated after LTP but depressed after LTD, indicating that mechanisms of EPSP amplification are regulated (see also Wang et al., 2003). Most importantly, the regulation of h-channel activity may constitute a major mechanism of expression. Apparent input resistance measured in the cell body is increased following induction of LTP induced by correlated activity or by high frequency stimulation (Campanac et al., 2008). This change is more pronounced when apical dendrites are recorded. In addition, the local decrease of the dendritic h-conductance with the use of dynamic-clamp techniques mimics EPSP-spike potentiation (Campanac et al., 2008). However, the regulation of A-type $\mathrm{K}^{+}$current in the dendrite (Frick et al., 2004; Kim et al., 2007) or the fast transient $\mathrm{Na}^{+}$current in the cell body (Xu et al., 2005) induced by positive correlation between synaptic activity and postsynaptic spiking may also facilitate EPSP-spike coupling in CA1 pyramidal neurons.

\section{SPATIAL AND TEMPORAL SELECTIVITY OF STDP-INDUCED INTRINSIC PLASTICITY SPATIAL SELECTIVITY}

The storage of information through the regulation of synaptic strength in models of memory is particularly attractive because of the property of input specificity. Given the large number of synapses per neuron $(>10000)$ and the huge number of neurons found in the brain $\left(10^{11}\right)$, a memory storage system that uses input-specific changes in synaptic transmission has a potentially enormous storage capacity. Although chemical synapses represent the major functional interface between neurons, following dendritic integration neuronal information is conveyed by action potentials. Thus, the activity-dependent regulation of ion channels located near the spike generation zone (the axon initial segment or the cell body) would change the throughput of all synapses that the neuron receives (Daoudal and Debanne, 2003; Zhang and Linden, 2003; Mozzachiodi and Byrne, 2010), possibly leading to a loss in the information stored at specific synapses. In this case, the specificity might be observed at the cellular level. Such global changes in neuronal excitability have been observed following brief episodes of synaptic (Aizenman and Linden, 2000; Sourdet et al., 2003) or spiking activity (Cudmore and Turrigiano, 2004; Paz et al., 2009) and in the presynaptic neuron when LTP or LTD is induced in hippocampal cells with spike-timing dependent protocols (Ganguly et al., 2000; Li et al., 2004). In these studies, neuronal excitability was assessed only in the cell body. Whether and to what extent intrinsic excitability changes in axonal and dendritic membranes remains unclear. Global changes have also been reported on the postsynaptic side when LTP is induced with correlated activity in CA1 pyramidal neurons ( $\mathrm{Xu}$ et al., 2005). However, several lines of evidence indicate that postsynaptic changes in intrinsic excitability induced by spike-timing dependent protocols are restricted to a localized region of the dendrites. Enhanced EPSP summation associated with synaptic potentiation is dependent on the position of the synaptic inputs (Wang et al., 2003). A-type $\mathrm{K}^{+}$channels are regulated in the dendrites of CA1 pyramidal neurons after induction of LTP (Frick et al., 2004). Potentiation and depression of dendritic integration observed in parallel with induction of spike-timing dependent 
synaptic plasticity are input specific (Campanac and Debanne, 2008). Finally, dendritic h-channel activity is locally reduced following induction of LTP (Campanac et al., 2008). Thus, intrinsic excitability changes associated with STDP-related protocols are localized in the dendrites of CA1 pyramidal neurons and may thus preserve the input specificity property for high-capacity information storage.

\section{TEMPORAL SELECTIVITY}

The temporal windows for synaptic potentiation and depression induced by correlated activity are generally not wider than a few tens of millisecond (Markram et al., 1997; Bi and Poo, 1998; Debanne et al., 1998; Feldman, 2000). They are usually precisely defined (at the millisecond scale) and depend on both the number of postsynaptic spikes (Debanne et al., 1994; Sourdet and Debanne, 1999) and dendritic location of the synaptic input (Froemke et al., 2005). Similar plasticity windows are observed for intrinsic plasticity. Presynaptic changes in neuronal excitability follow precisely the STDP rule (Li et al., 2004). At the postsynaptic side, EPSPspike plasticity also obeys the STDP learning rule (Campanac and Debanne, 2008). In these two examples, no changes in excitability at the pre- or postsynaptic side are observed out of the LTP and LTD time windows (i.e., for long positive or long negative intervals), suggesting common induction mechanisms for plasticity in synaptic efficacy and intrinsic excitability.

\section{FUNCTIONAL SYNERGY BETWEEN SYNAPTIC AND INTRINSIC PLASTICITY INDUCED BY STDP}

A functional synergy has been demonstrated for synaptic and intrinsic plasticity in central neurons at the pre- and postsynaptic side. LTP is generally associated with an increase in neuronal excitability (Armano et al., 2000; Ganguly et al., 2000; Daoudal et al., 2002; Wang et al., 2003; Xu et al., 2005; Campanac and Debanne, 2008; Campanac et al., 2008) whereas synaptic depression is usually accompanied by a reduced intrinsic excitability (Daoudal et al., 2002; Wang et al., 2003; Campanac and Debanne, 2008). Thus, plasticity in intrinsic excitability cannot be simply considered as an additional level of plasticity that makes understanding brain storage mechanisms more complex but rather, it can be incorporated into a general framework in which synaptic and intrinsic plasticity interact coherently (Daoudal and Debanne, 2003). This functional synergy insures significant changes in the neuronal output when synaptic plasticity is induced.

Functional synergy between synaptic and intrinsic plasticities might, however, be broken if the magnitude of synaptic plasticity is excessive and comes out of the physiological range (Debanne et al., 2003). This form of compensatory plasticity has been first studied theoretically in multi-compartment model of CA1 pyramidal neurons where intrinsic plasticity restored optimal firing range after synaptic potentiation (Siegel et al., 1994). In biological CA1 pyramidal neurons, synaptic potentiation of very large amplitude ( $~ 300 \%$ of the control EPSP) is accompanied by a decrease in excitability of CA1 pyramidal neurons (Fan et al., 2005). Conversely, nearly maximal synaptic depression $(-50 \%)$ is associated with an increase in excitability (Brager and Johnston, 2007). Both changes globally affect neuronal excitability, involve the regulation of h-channel activity, and are aimed to stabilize the overall neuronal activity by preventing excessive synaptic excitation. Therefore, these regulations are homeostatic in nature and compensate for excessive increases or decreases in synaptic excitation (Turrigiano and Nelson, 2004).

\section{CONCLUDING REMARIKS}

Temporal interactions of pre- and postsynaptic spiking in neural circuits bidirectionally regulate not only synaptic strength but also intrinsic excitability of pyramidal cells at both pre- and postsynaptic sides. At the postsynaptic side this plasticity is largely localized within the dendrites - involving the regulation of A-type $\mathrm{K}^{+}$and h-type cationic channels - and respects the input specificity of synaptic modifications, thus preserving high capacity of information storage. However, intrinsic plasticity is probably not synapse specific but rather may alter the excitability of dendritic branches over a few tens of micrometers (Losonczy et al., 2008). Additional experimental investigations will be necessary to precisely determine the spatial extent of intrinsic plasticity in branched neurons.

Synaptic activation of glutamate receptors is critical in the induction of long-term plasticity of intrinsic excitability in central neurons but one cannot exclude the contribution of other factors. Indeed, in most of the experiments reported so far (except the studies of Li et al., 2004; Losonczy et al., 2008), intrinsic plasticity is induced by stimulation of a large bundle of glutamatergic inputs and one cannot exclude stimulation of neuromodulators containing varicosities. In fact, neuromodulators are potent regulators of back-propagating action potentials (Hoffman and Johnston, 1999), ion channel activity (Cantrell and Catterall, 2001) and spike-timing dependent synaptic plasticity (Lin et al., 2003; Seol et al., 2007; Zhang et al., 2009). There are good reasons to believe that neuromodulators may also modulate induction of spiketiming dependent intrinsic plasticity. In addition, the gliotransmitter D-serine that is thought to be released by astrocytes upon neuronal stimulation (Mothet et al., 2005; review in Hamilton and Attwell, 2010) play a critical role in the induction of longterm synaptic plasticity (Henneberger et al., 2010). One cannot exclude that such new actors in interneuronal signaling may also play a major role in the induction of spike-timing dependent intrinsic plasticity.

Plasticity of intrinsic excitability is synergistic to synaptic plasticity in modulating neuronal output, thus enhancing the functional significance of synaptic modifications. However, compensatory mechanisms acting both at intrinsic and synaptic currents concur to maintain neuronal activity within physiological bounds (Siegel et al., 1994; Rumsay and Abbott, 2004; Rabinowitch and Segev, 2006). The synergy between synaptic and intrinsic plasticity might be broken outside this physiological range (Fan et al., 2005; Campanac et al., 2008). While learning rules for STDP of synaptic efficacy and intrinsic excitability are coordinated in pyramidal neurons, additional work is still needed to define the corresponding properties in GABAergic interneurons (Kullmann and Lamsa, 2007).

\section{ACKNOWLEDGMENTS}

This work is supported by CNRS, INSERM, ANR (Excion 2006 to Dominique Debanne), Région PACA (APO 2009 Plexin to Dominique Debanne), USNIH (NS36999 to Mu-Ming Poo). 


\section{REFERENCES}

Abbott, L. F., and Nelson, S. B. (2000). Synaptic plasticity: taming the beast. Nat. Neurosci. 3, 1178-1183.

Aizenman, C., and Linden, D. J. (2000). Rapid, synaptically driven increases in the intrinsic excitability of cerebellar deep nuclear neurons. Nat. Neurosci. 3, 109-111.

Alkon, D. L. (1984). Calcium-mediated reduction of ionic currents: a biophysical memory trace. Science 226, 1037-1045.

Andersen, P., Sundberg, S. H., Sveen, O., Swann, J.W., and Wigstöm, H. (1980). Possible mechanisms for long-lasting potentiation of synaptic transmission in hippocampal slices from guineapigs. J. Physiol. 302, 463-482.

Aou, S., Woody, C. D., and Birt, D. (1992). Increases in excitability of neurons of the motor cortex of cats after rapid acquisition of eye blink conditioning. J. Neurosci. 12, 560-569.

Armano, S., Rossi, P., Taglietti, V., and D’Angelo, E. (2000). Long-term potentiation of intrinsic excitability at the mossy fiber-granule cell synapse of rat cerebellum. J. Neurosci. 20, 5208-5216.

Bell, C., Han, V.Z., Sugawara, Y., and Grant, K. (1997). Synaptic plasticity in a cerebellum-like structure depends on temporal order. Nature 387, 278-281.

Bi, G. Q., and Poo, M. M. (1998). Synaptic modifications in cultured hippocampal neurons: dependence on spike timing, synaptic strength, and postsynaptic cell type. J. Neurosci. 18, 10484-10472.

Bliss, T. V. P., Lømo, T., and GardnerMedvin, A. R. (1973). "Synaptic plasticity in the hippocampal formation," in Macromolecules and Behaviour, eds G. B. Ansell and P. B. Bradley (London: MacMillan), 193-203.

Brager, D. H., and Johnston, D. (2007). Plasticity of intrinsic excitability during long-term depression is mediated through mGluR-dependent changes in Ih in hippocampal CA1 pyramidal neurons. J. Neurosci. 27, 13926-13937.

Campanac, E., Daoudal, G., Ankri, N., and Debanne, D. (2008). Down-regulation of dendritic Ih in CAl pyramidal neurons after LTP. J. Neurosci. 28, 8635-8643.

Campanac, E., and Debanne, D. (2008). Spike timing-dependent plasticity: a learning rule for dendritic integration in rat CAl pyramidal neurons. J. Physiol. 586, 779-793.

Cantrell,A. R., and Catterall, W.A. (2001). Neuromodulation of $\mathrm{Na}^{+}$channels: an unexpected form of cellular plasticity. Nat. Rev. Neurosci. 2, 397-407.

Carlier, E., Sourdet, V., Boudkkazi, S., Déglise, P., Ankri, N.
Fronzaroli-Molinieres, L., and Debanne, D. (2006). Metabotropic glutamate receptor subtype 1 regulates sodium currents in rat neocortical pyramidal neurons. J. Physiol. 577, 141-154.

Cudmore, R. H., and Turrigiano, G. G. (2004). Long-term potentiation of intrinsic excitability in LV visual cortical neurons. J. Neurophysiol. 92, 341-348.

Dan, Y., and Poo, M. M. (2006). Spike timing-dependent plasticity: from synapse to perception. Physiol. Rev. $86,1033-1048$

Daoudal, G., and Debanne, D. (2003). Long-term plasticity of intrinsic excitability: learning rules and mechanisms. Learn. Mem. 10, 456-465.

Daoudal, G., Hanada, Y., and Debanne, D. (2002). Bidirectional plasticity of excitatory postsynaptic potential (EPSP)spike coupling in CA1 hippocampal pyramidal neurons. Proc. Natl. Acad. Sci. U.S.A. 99, 14512-14517.

Debanne, D., Daoudal, G., Sourdet, V., and Russier, M. (2003). Brain plasticity and ion channels. J. Physiol. Paris 97, 403-414.

Debanne, D., Gähwiler, B. H., and Thompson, S. M. (1994). Asynchronous pre- and postsynaptic activity induces associative long-term depression in area CAl of the rat hippocampus in vitro. Proc. Natl. Acad. Sci. U.S.A. 91, 1148-1152.

Debanne, D., Gähwiler, B. H., and Thompson, S.M. (1996). Cooperative interactions in the induction of long-term potentation and depression of synaptic excitation between hippocampal CA3-CA1 cell pairs in vitro. Proc. Natl. Acad. Sci. U.S.A. 93, 11225-11230.

Debanne, D., Gähwiler, B. H., and Thompson, S. M. (1998). Long-term synaptic plasticity between pairs of individual CA3 pyramidal cells in rat hippocampal slice cultures. J. Physiol. 507, 237-247.

Disterhoft, J. F., Coulter, D. A., and Alkon, D. L. (1986). Conditioningspecific membrane changes of rabbit hippocampal neurons measured in vitro. Proc. Natl. Acad. Sci. U.S.A. 83, 2733-2737.

Du, J. L., Wei, H. P., Wang, Z. R., Wong, S. T., and Poo, M. M. (2009). Longrange retrograde spread of LTP and LTD from optic tectum to retina. Proc. Natl. Acad. Sci. U.S.A. 106, 1889-1896.

Egger, V., Feldmeyer, D., and Sakmann, B. (1999). Coincidence detection and changes of synaptic efficacy in spiny stellate neurons in rat barrel cortex. Nat. Neurosci. 2, 1098-1105.

Egorov, A. V., Hamam, B. N., Fransén, E., Hasselmo, M. E., and Alonso, A.
A. (2002). Graded persistent activity in entorhinal cortex neurons. Nature 420, 173-178.

Fan, Y., Fricker, D., Brager, D. H., Chen, X., Lu, H. C., Chitwood, R. A., and Johnston, D. (2005). Activitydependent decrease of excitability in rat hippocampal neurons through increases in Ih. Nat. Neurosci. 8 1542-1551.

Feldman, D. E. (2000). Timing-based LTP and LTD at vertical inputs to layer II/ III pyramidal cells in rat barrel cortex. Neuron 27, 45-56.

Fino, E., Glowinski, J., and Venance, L. (2005).Bidirectionalactivity-dependent plasticity at corticostriatal synapses. $J$ Neurosci. 25, 11279-11287.

Frick, A., Magee, J., and Johnston, D (2004). LTP is accompanied by an enhanced local excitability of pyramidal neuron dendrites. Nat. Neurosci. 7, 126-135.

Froemke, R. C., Poo, M. M., and Dan, Y (2005). Spike-timing-dependent synaptic plasticity depends on dendritic location. Nature 434, 221-225.

Ganguly, K., Kiss, L., and Poo, M. M. (2000). Enhancement of presynaptic neuronal excitability by correlated presynaptic and postsynaptic spiking. Nat. Neurosci. 3, 1018-1026.

Gustafsson, B., Wigström, H., Abraham W. C., and Huang, Y.Y. (1987). Longterm potentiation in the hippocampus using depolarizing current pulses as the conditioning stimulus to single volley synaptic potentials. J. Neurosci 7, 774-780.

Hamilton, N. B., and Attwell, D. (2010) Do astrocytes really exocytose neurotransmitters? Nat. Rev. Neurosci. 11 227-238.

Hebb, D. O. (1949). The Organization of Behaviour. New York, NY: Wiley.

Henneberger, C., Papouin, T., Oliet, S. H., and Rusakov, D. A. (2010). Longterm potentiation depends on release of D-serine from astrocytes. Nature 463, 232-236.

Hoffman, D. A., and Johnston, D. (1999) Neuromodulation of dendritic action potentials. J. Neurophysiol. 81 , 408-441.

Ireland, D. R., and Abraham, W.C. (2002) Group I mGluRs increase excitability of hippocampal CA1 pyramidal neurons by a PLC-independent mechanism. J. Neurophysiol. 88, 107-116.

Jacob, V., Brasier, D. J., Erchova, I. Feldman, D., and Shulz, D. E. (2007). Spike timing-dependent synaptic depression in the in vivo barrel cortex of the rat. J. Neurosci. 27, 1271-1284.

Kim, J., Jung, S.C., Clemens, A. M., Petralia, R. S., and Hoffman, D. A. (2007) Regulation of dendritic excitability by activity-dependent trafficking of the A-type $\mathrm{K}+$ channel subunit $\mathrm{Kv} 4.2$ in hippocampal neurons. Neuron 54 933-947.

Kim, S. J., and Linden, D. J. (2007). Ubiquitous plasticity and memory storage. Neuron 56, 582-592.

Kullmann, D. M., and Lamsa, K. P. (2007) Long-term synaptic plasticity in hippocampal interneurons. Nat. Rev. Neurosci. 8, 687-699.

Levy, W. B., and Steward, O. (1983). Temporal contiguity requirements for long-term associative potentiation/depression in the hippocampus. Neuroscience 8, 791-797.

Li, C. Y., Lu, J. T., Wu, C. P., Duan, S. M., and Poo, M. M. (2004). Bidirectional modification of presynaptic neuronal excitability accompanying spike timing-dependent synaptic plasticity. Neuron 41, 257-268.

Lin, Y. W., Min, M. Y., Chiu, T. H., and Yang, H. W. (2003). Enhancement of associative long-term potentiation by activation of $\beta$-adrenergic receptors at CAl synapses in rat hippocampal slices. J. Neurosci. 23, 4173-4181.

Losonczy, A., Makara, J. K., and Magee, J. C. (2008). Compartmentalized dendritic plasticity and input feature storage in neurons. Nature 452, 436-441.

Markram, H., Lübke, J., Frotscher, M. and Sakmann, B. (1997). Regulation of synaptic efficacy by coincidence of postsynaptic APs and EPSPs. Science 275, 213-215.

Matthews, E. A., Weible, A. P., Shah, S., and Disterhoft, J. F. (2008). The BK-mediated fAHP is modulated by learning a hippocampus-dependent task. Proc. Natl. Acad. Sci. U.S.A. 105, 15154-15159.

Mehta, M. R., Quirk, M. C., and Wilson, M. A. (2000). Experience-dependent asymmetric shape of hippocampal receptive fields. Neuron 25, 707-715.

Melyan, Z., Lancaster, B., and Wheal, $\mathrm{H}$. V. (2004). Metabotropic regulation of intrinsic excitability by synaptic activation of kainate receptors. J. Neurosci. 24, 4530-4534.

Mothet, J. P., Pollegioni, L., Ouanounou, G., Martineau, M., Fossier, P., and Baux, G. (2005). Glutamate receptor activation triggers a calcium-dependent and SNARE protein-dependent release of the gliotransmitter D-serine.Proc. Natl. Acad. Sci. U.S.A. 102, 5606-5611.

Moyer, J. R., Thompson, L. T., and Disterhoft, J. F. (1996). Trace eyeblink conditioning increases CAl excitability in a transient and learning-specific manner. J. Neurosci. 16, 5536-5546.

Mozzachiodi, R., and Byrne, J. H. (2010). More than synaptic plasticity: role of nonsynaptic plasticity in learning and memory. Trends Neurosci. 33, 17-26.

Nelson, A. B., Krispel C. M., Sekimjak, C and du Lac, S. (2003). Long-lasting 
increases in intrinsic excitability triggered by inhibition. Neuron 40, 609-620.

Oh, M. M., Kuo, A. G., Wu, W. W., Sametsky, E. A., and Disterhoft, J. F. (2003). Watermaze learning enhances excitability of CA1 pyramidal neurons. J. Neurophysiol. 90, 2171-2179.

Pawlak, V., and Kerr J. N. D. (2008). Dopamine receptor activation is required for corticostriatal spiketiming-dependent plasticity. $J$. Neurosci. 28, 2435-2446.

Paz, J. T., Mahon, S., Tiret, P., Genet, S., Delord, B., and Charpier, S. (2009). Multiple forms of activity-dependent intrinsic plasticity in layer $\mathrm{V}$ cortical neurones in vivo. J. Physiol. 587, 3189-3205.

Rabinowitch, I., and Segev, I. (2006). The endurance and selectivity of spatial patterns of long-term potentiation/ depression in dendrites under homeostatic synaptic plasticity. J. Neurosci. 26, 13474-13484.

Ruiz, A., Sachdhanandam, S., Utvik, J. K., Coussen, F., and Mulle, C. (2005). Distinct subunits in heteromeric kainite receptors mediate ionotropic and metabotropic function at hippocampal mossy fiber synapses. J. Neurosci. 25, 11710-11718.

Rumsay C. C., and Abbott L. F. (2004). Equalization of synaptic efficacy by activity- and timing-dependent synaptic plasticity. J. Neurophysiol. 91, 2273-2280.

Saar, D., Grossman, Y., and Barkai, E. (1998). Reduced afterhyperpolarization in rat piriform cortex pyramidal neurons is associated with increased learning capability during operant conditioning. Eur. J. Neurosci. 10, 1518-1523.

Scholz, K. P., and Byrne, J. H. (1987). Long-term sensitization in Aplysia: biophysical correlates in tail sensory neurons. Science 235, 685-687.
Schreurs, B. G., Tomsic, D., Gusev, P. A., and Alkon, D. L. (1997). Dendritic excitability microzones and occluded long-term depression after classical conditioning of the rabbit's nictitating membrane response. J. Neurophysiol. 77, 86-92.

Schuett, S., Bonhoeffer, T., and Hübener M. (2001). Pairing-induced changes of orientation maps in cat visual cortex. Neuron 32, 325-337.

Seol, G.H.,Ziburkus, J.,Huang, S.Y., Song, L., Kim, I. T., Takamiya, K., Huganir, R. L., Lee, H. K., and Kirkwood, A. (2007). Neuromodulators control the polarity of spike-timing-dependent synaptic plasticity. Neuron 55, 919-929.

Siegel, M., Marder, E., and Abbott, L. F. (1994). Activity-dependent current distribution in model neurons. Proc. Natl. Acad. Sci. U.S.A. 91, 11308-11312.

Sjöström, P. J., Rancz, E. A., Roth, A., and Häusser, M. (2008). Dendritic excitability and synaptic plasticity. Physiol. Rev. 88, 768-840.

Sjöström, P. J., Turrigiano, G. G., and Nelson, S. B. (2001). Rate, timing, and cooperativity jointly determine cortical synaptic plasticity. Neuron 32, 1149-1164.

Song, S., Miller, K. D., and Abbott, L. F. (2000). Competitive Hebbian learning through spike-timing-dependent synaptic plasticity. Nat. Neurosci. 3, 919-926.

Sourdet, V., and Debanne, D. (1999). The role of dendritic filtering in associative long-term synaptic plasticity. Learn. Mem. 6, 422-447.

Sourdet, V., Russier, M., Daoudal, G., Ankri, N., and Debanne, D. (2003). Long-term enhancement of neuronal excitability and temporal fidelity mediated by metabotropic glutamate receptor subtype 5. J. Neurosci. 23, 10238-10248.
Spruston, N. (2008). Pyramidal neurons: dendritic structure and synaptic integration. Nat. Rev. Neurosci. 9, 206-221.

Stent, G. S. (1973). A physiological mechanism for Hebb's postulate of learning. Proc. Natl. Acad. Sci. U.S.A. 70, 997-1001.

Tao, H., Zhang, L. I., Bi, G. Q., and Poo, M M. (2000). Selective presynaptic propagation of long-term potentiation in defined neural networks. J. Neurosci. 20, 3233-3243.

Turrigiano, G. G., and Nelson, S. B. (2004) Homeostatic plasticity in the developing nervous system. Nat. Rev. Neurosci. 5, 97-107.

Tzounopoulos, T., Kim, Y., Oertel, D., and Trussell, L. O. (2004). Cell-specific, spike timing-dependent plasticities in the dorsal cochlear nucleus. Nat. Neurosci. 7, 719-725.

Wang, S. S. H., Denk, W., and Häusser, M. (2000). Coincidence detection in single dendritic spines mediated by calcium release. Nat. Neurosci. 3 1266-1273.

Wang, Z., Xu, N. I., Wu, C. P., Duan, S. and Poo, M. M. (2003). Bidirectional changes in spatial dendritic integration accompanying long-term synaptic modifications. Neuron 37, 463-472.

Wolters, A., Sandbrink, F., Schlottmann, A., Kunesch, E., Stefan, K., Cohen, L. G., Benecke, R., and Classen, J. (2003). A temporally asymmetric Hebbian rule governing plasticity in the human motor cortex. J. Neurophysiol. 89 2339-2345.

Xu, J., Kang, N., Jiang, L., Nedergaard, M., and Kang, J. (2005). Activitydependent long-term potentiation of intrinsic excitability in hippocampal CA1 pyramidal neurons. J. Neurosci. 25, 1750-1760.

Xu, N.L., Ye, C. Q., Poo, M. M., and Zhang, X. H. (2006). Coincidence detection of synaptic inputs is facilitated at the distal dendrites after long-term potentiation induction. J. Neurosci. 26, 3002-3009.

Yao, H., and Dan, Y. (2001). Stimulus timing-dependent plasticity in cortical processing of orientation. Neuron 32, 315-323.

Zhang, J. C., Lau, P. M., and Bi, G. Q. (2009). Gain in sensitivity and loss in temporal contrast of STDP by dopaminergic modulation at hippocampal synapses. Proc. Natl. Acad. Sci. U.S.A. 106, 13028-13033.

Zhang, L. I., Tao, H. W., Holt, C. E., Harris, W.A., and Poo, M.M. (1998).A critical window for cooperation and competition among developing retinotectal synapses. Nature 395, 37-44.

Zhang, W., and Linden, D. J. (2003). The other side of the engram: experiencedriven changes in neuronal intrinsic excitability. Nat. Rev. Neurosci. 4, 885-900.

Conflict of Interest Statement: The authors declare that the research was conducted in the absence of any commercial or financial relationships that could be construed as a potential conflict of interest.

Received: 29 January 2010; paper pending published: 16 February 2010; accepted: 30 May 2010; published online: 18 June 2010.

Citation: Debanne D and Poo M-M (2010) Spike-timing dependent plasticity beyond synapse - pre- and post-synaptic plasticity of intrinsic neuronal excitability. Front. Syn. Neurosci. 2:21. doi: 10.3389/ fnsyn.2010.00021

Copyright $($ C 2010 Debanne and Poo. This is an open-access article subject to an exclusive license agreement between the authors and the Frontiers Research Foundation, which permits unrestricted use, distribution, and reproduction in any medium, provided the original authors and source are credited. 